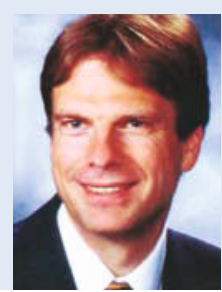

Prof.

Dr. med.

Thomas

Lehrnbecher,

Frankfurt

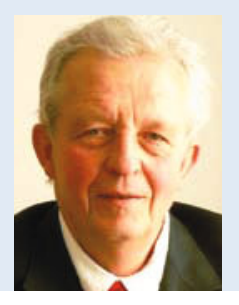

Prof.

Dr. med. Kurt

Malberg,

Dresden-

Loschwitz

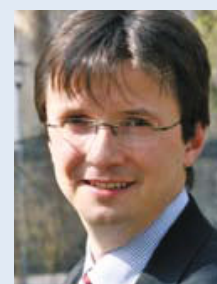

Prof.

Dr. med.

Peter

Schwarz,

Dresden

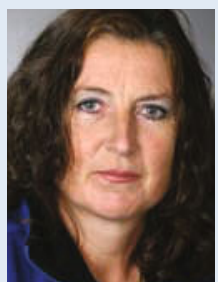

Dr. med.

Kirsten

Stollhoff,

Hamburg

\section{Auffrischimpfungen nach Chemotherapie - Wunsch und Wirklichkeit}

Nach Beendigung einer Chemotherapie sollten krebskranke Kinder gemäß internationaler Standards Auffrischimpfungen erhalten. Ein australisches Forscherteam untersuchte, wie viele Kinder wirklich entsprechend der australischen Empfehlungen geimpft werden.

$\mathrm{D}$ urch eine Chemotherapie wird das Immunsystem mit B- und T-Lymphozyten stark unterdrückt, was bei einem beträchtlichen Teil der Krebspatienten zu einem Verlust der vor der Krankheit erworbenen Impfantikörper führt. Deswegen werden für Kinder nach Chemotherapie Auffrischimpfungen empfohlen. Dabei sollen im Allgemeinen Totimpfungen etwa sechs und Lebendimpfungen etwa neun Monate nach Beendigung der Chemotherapie verabreicht werden. Allerdings sind noch viele Fragen hinsichtlich der Impfstrategie nach Chemotherapie offen, so z. B., ob auch Kinder mit relativ geringer Therapieintensität (wie z. B. leichte Formen einer Langerhanszell-Histiozytose oder niedrige Stadien eines Nephroblastoms) eine Auffrischimpfung benötigen. Umgekehrt besteht bei vielen Eltern die (unbegründete) Angst, dass eine Impfung eine Krebserkrankung auslösen oder reaktivieren könnte.

Wissenschaftler aus Australien wollten wissen, wie es um die Auffrischimpfungen steht. Einschlusskriterien für ihre Studie war ein Alter der Patienten zwischen 0 und 18 Jahre und ein Mindestabstand zur Chemotherapie von sechs Monaten. In die Analyse gingen insgesamt 89 Patienten ein. Der Altersmedian bei Diagnosestellung lag bei 5,3
Jahren, wobei $56 \%$ der Patienten an einer Leukämie und 44\% der Patienten an einem soliden Tumor litten. Der mediane Abstand zur Beendigung der Chemotherapie lag bei 3,1 Jahren. Nach Analyse aller verfügbaren Informationsquellen erhielten knapp 40\% (35/89) der Patienten nach Beendigung der Chemotherapie keine Auffrischimpfung. Jüngere Patienten sowie Patienten mit der Diagnose Leukämie hatten eine signifikant höhere Rate von Auffrischimpfungen.

Die Autoren schlussfolgern, dass die schlechte Compliance für Auffrischimpfungen nach Chemotherapie unbedingt verbessert werden muss. Die könnte zum Beispiel durch Forschungsaktivitäten erreicht werden, die eine bessere Evidenz für den Nutzen der bestehenden Empfehlungen liefern. Weiterhin müssen sowohl die betreuenden Ärzte als auch die betroffenen Kinder und deren Eltern von dem Nutzen der Auffrischimpfungen überzeugt werden.

Kommentar: Die Ergebnisse dieser Arbeit zeigen eindrucksvoll, wie unbefriedigend die Nachbetreuung von krebskranken Kindern in einigen Teilaspekten immer noch ist. Wichtig wäre es nun, die Gründe für diese Probleme herauszufinden. So müsste geklärt werden, wer die Kinder hinsichtlich der Impffragen nach Chemotherapie berät (pädiatrischer Hämatologe/Onkologe versus Kinderarzt vor Ort) und wie diese Beratung in der Realität aussieht (Impfrage wird nicht besprochen versus $\mathrm{Zu}$ - oder Abraten). Weiterhin müssen gut konzipierte Studien zu Impfungen nach Chemotherapie durchgeführt werden, die sowohl den betreuenden Ärzten als auch den Eltern betroffener Patienten den Sinn der empfohlenen Maßnahmen zeigen.

Prof. Dr. Thomas Lehrnbecher

Crawford et al. Survivors of childhood cancer: an Australian audit of vaccination status after treatment. Pediatr Blood Cancer 2010; 54: 128-33 